\title{
Postmastectomy Radiotherapy in Patients with pT1-2N1 Breast Cancer Treated with Taxane-Based Chemotherapy: A Retrospective Multicenter Analysis (KROG 1418)
}

\section{Yeon-Joo Kim, MD \\ Won Park, MD2 \\ Boram Ha, MD ${ }^{1}$ \\ Boram Park, $\mathrm{MS}^{3}$ \\ Jungnam Joo, $\mathrm{PhD}^{3}$ \\ Tae Hyun Kim, MD ${ }^{1}$ \\ In Hae Park, MD ${ }^{1}$ \\ Keun Seok Lee, MD ${ }^{1}$ \\ Eun Sook Lee, MD' \\ Kyung Hwan Shin, MD ${ }^{4}$ \\ Haeyoung Kim, MD 5 \\ Jeong II Yu, MD2 \\ Doo Ho Choi, MD2 \\ Seung Jae Huh, MD² \\ Chan Woo Wee, MD ${ }^{4}$ \\ Kyubo Kim, MD ${ }^{6}$ \\ Kyung Ran Park, MD \\ Yong Bae Kim, MD \\ Sung Ja Ahn, MD \\ Jong Hoon Lee, MD 9 \\ Jin Hee Kim, MD ${ }^{10}$ \\ Mison Chun, MD ${ }^{11}$ \\ Hyung-Sik Lee, MD ${ }^{12}$ \\ Jung Soo Kim, MD'13 \\ Jihye Cha, MD ${ }^{14}$}

${ }^{*} A$ list author's affiliations appears at the end of the paper.

\section{Purpose}

The purpose of this study was to evaluate the impact of postmastectomy radiotherapy (PMRT) on loco-regional recurrence-free survival (LRRFS), disease-free survival (DFS), and overall survival (OS) in pT1-2N1 patients treated with taxane-based chemotherapy.

\section{Materials and Methods}

We retrospectively reviewed the medical data of pathological N1 patients who were treated with modified radical mastectomy and adjuvant taxane-based chemotherapy in 12 hospitals between January 2006 and December 2010.

\section{Results}

We identified 714 consecutive patients. The median follow-up duration was 69 months (range, 1 to 114 months) and the 5-year LRRFS, DFS, and OS rates were 97\%, 94\%, and $98 \%$, respectively, in patients who received PMRT (PMRT [+]). The corresponding figures were 96\%, 90\%, and 96\%, respectively, in patients who did not receive PMRT (PMRT [-]). PMRT had no significant impact on survival. Upon multivariable analysis, only the histological grade $(\mathrm{HG})$ was statistically significant as a prognostic factor for LRRFS and DFS. In a subgroup analysis of HG 3 patients, PMRT $(+)$ showed better DFS $(p=0.081)$.

\section{Conclusion}

PMRT had no significant impact on LRRFS, DFS, or OS in pT1-2N1 patients treated with taxane-based chemotherapy. PMRT showed a marginal benefit for DFS in HG 3 patients. Randomized studies are needed to confirm the benefit of PMRT in high risk patients, such as those with HG 3.

\author{
Correspondence: Won Park, MD \\ Department of Radiation Oncology, \\ Samsung Medical Center, \\ Sungkyunkwan University School of Medicine, \\ 81 Irwon-ro, Gangnam-gu, Seoul 06351, Korea \\ Tel: 82-2-3410-2616 \\ Fax: 82-2-3410-2619 \\ E-mail: wonro.park@samsung.com \\ Received October 21, 2016 \\ Accepted December 13, 2016 \\ Published Online December 26, 2016
}

Key words

Breast neoplasms, Radiotherapy, Taxane, Recurrence, Disease-free survival, Survival 


\section{Introduction}

The National Comprehensive Cancer Network's clinical practice guidelines for breast cancer strongly recommend that postmastectomy radiotherapy (PMRT) be considered for patients with early-stage breast cancer with 1-3 positive axillary nodes [1]. The British Columbia randomized trial presented better survival rates for patients that received chemotherapy and radiation therapy than chemotherapy alone in lymph node (LN)-positive breast cancer treated by modified radical mastectomy (MRM) [2]. A subgroup analysis by the Danish Breast Cancer Cooperative Group (DBCG) $82 \mathrm{~B} \& \mathrm{C}$ randomized trial also showed a survival benefit after PMRT in patients with 1-3 as well as 4+ positive LNs [3]. A meta-analysis of 22 randomized trials confirmed that PMRT reduced loco-regional recurrence (LRR), overall recurrence, and breast cancer mortality in patients with positive LNs [4].

However, the majority of chemotherapy regimens of these studies used CMF (cyclophosphamide, methotrexate, and 5 -fluorouracil), which is less effective than modern taxanebased chemotherapies [5-7]. To date, the benefit of PMRT after taxane-based chemotherapy in pT1-2N1 patients remains unclear.

Therefore, we conducted this study to evaluate the impact of PMRT on loco-regional recurrence-free survival (LRRFS), disease-free survival (DFS), and overall survival (OS) in pT12N1 patients treated with taxane-based chemotherapy.

\section{Materials and Methods}

\section{Patients}

We retrospectively reviewed and collected the medical data of $\mathrm{pN} 1$ breast cancer patients who were treated with MRM and adjuvant taxane-based chemotherapy in 12 hospitals in Korea between January 2006 and December 2010. This retrospective observational study was approved by the Institutional Review Board of each hospital.

The inclusion criteria were $\mathrm{pN} 1$ breast cancer patients, completion of planned chemotherapy, and information available regarding the pathologic features of tumors, including hormone receptor status. We excluded patients who received neo-adjuvant chemotherapy, previous chest wall radiotherapy, were pT3-4 or were male. Overall, we identified 714 consecutive patients.

We collected pathologic features of tumors, such as tumor size, resection margin (RM), lymphovascular invasion (LVI), histologic grade (HG), estrogen receptor (ER), progesterone receptor (PR), human epidermal growth factor receptor 2 (HER2) status, Ki-67, number of positive LNs, and extracapsular extension (ECE). The ER and PR positivity were defined as any positive nuclear staining. HER2 positivity was defined as an immunohistochemistry score of $3+$ or $2+$ along with fluorescent in situ hybridization-positive results or chromogenic in situ hybridization-positive results for HER2 gene amplification. Molecular subtypes of breast cancer were categorized as follows: (1) luminal A: ER+ and/or PR+ and HER2-, (2) luminal B: ER+ and/or PR+ and HER2+, (3) HER2: ER- and PR- and HER2+, and (4) triple negative: ERand PR- and HER2-.

\section{Treatments}

All 714 patients underwent MRM, and a majority (94.8\%) of these patients received axillary LN dissection. Adjuvant radiotherapy was conducted according to the physician's preference and institutional policy. In addition to the chest wall, radiation was delivered to the supraclavicular (SCL) and / or internal mammary node (IMN) area in some patients according to the radiation oncologist's preference. Trastuzumab therapy was indicated for patients with HER2-overexpressing tumors, while hormone therapy was indicated for patients with positive hormonal receptors.

PMRT was performed in 130 patients (18.2\%). In addition to chest wall radiotherapy, SCL radiotherapy was conducted in 99 patients $(76.2 \%, 99 / 130)$. Among these patients, 61 $(46.9 \%, 61 / 130)$ also received IMN radiotherapy. The median radiation doses to the chest wall, SCL and IMN were 50.4 Gy/28 fractions (range, 45 Gy/25 fractions to 50.4 Gy/ 28 fractions). Chest wall irradiation was conducted with 4- or 6-MV photon beams of two tangential fields that covered the chest wall and axillary LN level I. SCL field was treated with 4- or 6-MV photon beams with or without posterior axillary boost and covered axillary LN level II-III and SCL LN. IMN radiotherapy techniques, which were determined at the discretion of the physician, included the reverse hockey stick, standard tangent, partial wide tangent, and photon/electron combination techniques.

Most patients (95.8\%) were treated with AC (doxorubicin and cyclophosphamide)-T (taxane), while the rest received other taxane-based chemotherapies, such as TC (docetaxel and cyclophosphamide)-T (2.4\%), FAC (fluorouracil [5-FU], doxorubicin, and cyclophosphamide)/FEC (5-FU, epirubicin, and cyclophosphamide)-T (0.4\%), TAC (docetaxel, doxorubicin, and cyclophosphamide) or TAC-T (0.3\%), EC (epirubicin and cyclophosphamide)-T $(0.1 \%)$ and others (1\%). Trastuzumab and hormones were given to 104 patients (14.6\%) who were HER2-positive and 558 patients (78.2\%) who were hormonal receptor-positive, respectively. 
Table 1. Patient and tumor characteristics $(n=714)$

\begin{tabular}{|c|c|}
\hline Characteristic & No. $(\%)$ \\
\hline Age, median (range, yr) & $48(24-74)$ \\
\hline \multicolumn{2}{|l|}{ Pathology } \\
\hline Ductal & $667(93.4)$ \\
\hline Lobular & $27(3.8)$ \\
\hline Micropapillary & $7(1.0)$ \\
\hline Mixed & $6(0.8)$ \\
\hline Mucinous & $5(0.7)$ \\
\hline Medullary & $1(0.1)$ \\
\hline Other & $1(0.1)$ \\
\hline \multicolumn{2}{|l|}{ T classification } \\
\hline 1 & $291(40.8)$ \\
\hline 2 & $423(59.2)$ \\
\hline \multicolumn{2}{|l|}{ Resection margin } \\
\hline Negative & $701(98.2)$ \\
\hline Positive & $8(1.1)$ \\
\hline $\mathrm{N} / \mathrm{A}$ & $5(0.7)$ \\
\hline \multicolumn{2}{|l|}{ Lymphatic and vascular invasion } \\
\hline Negative & $421(59.0)$ \\
\hline Positive & $273(38.2)$ \\
\hline $\mathrm{N} / \mathrm{A}$ & $20(2.8)$ \\
\hline \multicolumn{2}{|l|}{ Histologic grade } \\
\hline 1 & $85(11.9)$ \\
\hline 2 & $311(43.6)$ \\
\hline 3 & $305(42.7)$ \\
\hline $\mathrm{N} / \mathrm{A}$ & $13(1.8)$ \\
\hline \multicolumn{2}{|l|}{ Estrogen hormonal receptor } \\
\hline Negative & 149 (20.9) \\
\hline Positive & $548(76.8)$ \\
\hline $\mathrm{N} / \mathrm{A}$ & $17(2.4)$ \\
\hline \multicolumn{2}{|l|}{ Progesterone hormonal receptor } \\
\hline Negative & $206(28.9)$ \\
\hline Positive & $487(68.2)$ \\
\hline $\mathrm{N} / \mathrm{A}$ & $21(2.9)$ \\
\hline \multicolumn{2}{|l|}{ HER2/neu proto-oncogene } \\
\hline Negative & $530(74.2)$ \\
\hline Positive & $184(25.8)$ \\
\hline \multicolumn{2}{|l|}{ Molecular subtype } \\
\hline Luminal A & $469(65.7)$ \\
\hline Luminal B & $98(13.7)$ \\
\hline HER2 & $80(11.2)$ \\
\hline Triple negative & $55(7.7)$ \\
\hline $\mathrm{N} / \mathrm{A}$ & $12(1.7)$ \\
\hline \multicolumn{2}{|l|}{ Ki-67 (\%) } \\
\hline Negative & $3(0.4)$ \\
\hline$\leq 15$ & $399(55.9)$ \\
\hline $16-30$ & $141(19.7)$ \\
\hline$>30$ & $131(18.3)$ \\
\hline N/A & $40(5.6)$ \\
\hline Total dissected lymph node, median (range) & $16(1-55)$ \\
\hline
\end{tabular}

Table 1. Continued

\begin{tabular}{|lc}
\hline Characteristic & No. $(\%)$ \\
\hline $\begin{array}{l}\text { Positive lymph node } \\
1\end{array}$ & $399(55.9)$ \\
2 & $202(28.3)$ \\
\hline 3 & $111(15.5)$ \\
\hline N/A & $2(0.3)$ \\
\hline Extracapsular extension & $330(46.2)$ \\
\hline Negative & $272(38.1)$ \\
\hline Positive & $112(15.7)$ \\
\hline N/A & \\
\hline N/A, not available; HER2, human epidermal growth factor \\
receptor 2.
\end{tabular}

\section{Endpoints}

LRR was defined as ipsilateral chest wall, axilla, IMN and SCL LN recurrence. All survival endpoints were calculated from the date of surgery. DFS was defined as the time from the date of surgery to the date of any recurrence. OS was defined as the time from the date of surgery to the date of death from any reason.

\section{Statistical Analyses}

The patient and treatment characteristics were summarized as the median (range) values for continuous variables, and frequency (percentage) values for categorical variables. The difference in distributions according to the PMRT group (PMRT [+] vs. PMRT [-]) was tested using Pearson's chisquared test or Fisher exact test. The multivariable Cox proportional hazard model was fitted to analyze the effect of PMRT on patient survival while adjusting for the effects of other variables. The backward variable selection with an elimination criterion of $\mathrm{p}>0.1$ was applied to fit the multivariable model. In addition, since distributions of several variables were significantly different between PMRT groups, we conducted propensity score matching to balance these differences before evaluating the effects of PMRT on patient survival. The propensity score was first estimated using the logistic regression, where the outcome variable was PMRT $(+)$ or PMRT $(-)$, and the explanatory variables were those that were significantly imbalanced between PMRT groups. Next, based on the estimated propensity score, two patients in the PMRT (-) group were matched to each PMRT (+) patient. The survival curves were estimated using the Kaplan-Meier method, and the log-rank test was performed to test the survival difference for this matched dataset. p-val- 
Table 2. Comparisons of patient and treatment characteristics between PMRT (-) and PMRT (+)

\begin{tabular}{|c|c|c|c|c|}
\hline Variable & $\begin{array}{c}\text { Total } \\
(n=714)\end{array}$ & $\begin{array}{c}\text { PMRT (-) } \\
(\mathrm{n}=584)\end{array}$ & $\begin{array}{c}\text { PMRT (+) } \\
(n=130)\end{array}$ & p-value \\
\hline \multicolumn{5}{|l|}{ Age (yr) } \\
\hline$<40$ & 131 & $107(18.3)$ & $24(18.5)$ & $0.970^{\mathrm{a})}$ \\
\hline$\geq 40$ & 583 & $477(81.7)$ & $106(81.5)$ & \\
\hline \multicolumn{5}{|l|}{ Pathology } \\
\hline IDC & 667 & $553(94.7)$ & $114(87.7)$ & $0.004^{\mathrm{a})}$ \\
\hline Non-IDC & 47 & $31(5.3)$ & $16(12.3)$ & \\
\hline \multicolumn{5}{|l|}{ T classification } \\
\hline 1 & 291 & $239(40.9)$ & $52(40.0)$ & $0.846^{\mathrm{a})}$ \\
\hline 2 & 423 & $345(59.1)$ & $78(60.0)$ & \\
\hline \multicolumn{5}{|c|}{ Resection margin (miss $=5$ ) } \\
\hline Negative & 701 & $578(99.8)$ & $123(94.6)$ & $<0.001^{\mathrm{b})}$ \\
\hline Positive & 8 & $1(0.2)$ & $7(5.4)$ & \\
\hline \multicolumn{5}{|c|}{ Lymphatic and vascular invasion $($ miss $=20$ ) } \\
\hline Negative & 421 & $363(63.2)$ & $58(48.3)$ & $0.002^{\mathrm{a})}$ \\
\hline Positive & 273 & $211(36.8)$ & $62(51.7)$ & \\
\hline \multicolumn{5}{|c|}{ Histologic grade (miss=13) } \\
\hline $1-2$ & 396 & $314(54.7)$ & $82(64.6)$ & $0.043^{\mathrm{a})}$ \\
\hline 3 & 305 & $260(45.3)$ & $45(35.4)$ & \\
\hline \multicolumn{5}{|c|}{ Molecular subtype (miss=12) } \\
\hline Luminal A & 469 & $383(66)$ & $86(70.5)$ & $0.342^{\mathrm{a})}$ \\
\hline Nonluminal A & 233 & $197(34)$ & $36(29.5)$ & \\
\hline \multicolumn{5}{|c|}{ Ki-67 (\%) (miss=40) } \\
\hline$\leq 15$ & 402 & $331(59.9)$ & $71(58.7)$ & $0.811^{\mathrm{a})}$ \\
\hline$>15$ & 272 & $222(40.1)$ & $50(41.3)$ & \\
\hline \multicolumn{5}{|c|}{ Total dissected lymph node } \\
\hline$<10$ & 109 & $84(14.4)$ & $25(19.2)$ & 0.166 \\
\hline$\geq 10$ & 605 & $500(85.6)$ & $105(80.8)$ & \\
\hline \multicolumn{5}{|c|}{ Positive lymph node (miss=3) } \\
\hline 1 & 398 & $343(58.9)$ & $55(42.6)$ & $<0.001^{\text {a) }}$ \\
\hline $2-3$ & 313 & $239(41.1)$ & $74(57.4)$ & \\
\hline \multicolumn{5}{|c|}{ Extracapsular extension (miss=112) } \\
\hline Negative & 330 & $286(53.9)$ & $44(62.0)$ & $0.197^{\mathrm{a})}$ \\
\hline Positive & 272 & $245(46.1)$ & $27(38.0)$ & \\
\hline
\end{tabular}

Values are presented as number (\%). PMRT, postmastectomy radiotherapy; IDC, invasive ductal carcinoma. ${ }^{a}$ Pearson chi-

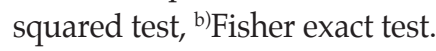

ues of $<0.05$ were considered statistically significant, and all statistical analyses were performed using the SAS ver. 9.4 (SAS Institute Inc., Cary, NC) and R ver. 3.2.3 programs.

\section{Results}

\section{Patient and tumor characteristics}

The patient and tumor characteristics are shown in Table 1. The median age was 48 years (range, 24 to 74 years), and the majority of patients had ductal carcinoma (93.4\%). The RM and LVI data were positive in $1.1 \%$ and $38.2 \%$ of patients, respectively. The HG was 3 in 305 patients (42.7\%). 
Table 3. Patterns of first failure

\begin{tabular}{|c|c|c|c|}
\hline Variable & $\begin{array}{c}\text { PMRT (-) } \\
(\mathrm{n}=584)\end{array}$ & $\begin{array}{c}\text { PMRT (+) } \\
(\mathbf{n}=130)\end{array}$ & $\begin{array}{c}\text { Total } \\
(n=714)\end{array}$ \\
\hline Local recurrence & $6(1.0)$ & $1(0.8)$ & $7(1.0)$ \\
\hline Regional recurrence & $22(3.8)$ & $4(3.1)$ & $26(3.6)$ \\
\hline AXL & $7(1.2)$ & $1(0.8)$ & $8(1.1)$ \\
\hline SCL & $5(0.9)$ & $2(1.5)$ & $7(1.0)$ \\
\hline IMN & $6(1.0)$ & $1(0.8)$ & $7(1.0)$ \\
\hline AXL and SCL & $2(0.3)$ & 0 & $2(0.3)$ \\
\hline AXL and IMN & 0 & 0 & 0 \\
\hline SCL and IMN & $1(0.2)$ & 0 & $1(0.1)$ \\
\hline AXL and SCL and IMN & $1(0.2)$ & 0 & $1(0.1)$ \\
\hline Distant metastasis & $48(8.2)$ & $6(4.6)$ & $54(7.6)$ \\
\hline
\end{tabular}

Values are presented as number $(\%)$. Multiple locations of recurrence may have been observed in a patient. PMRT, postmastectomy radiotherapy; AXL, axillary; SCL, supraclavicular; IMN, internal mammary.

Table 2 compares the patient and treatment characteristics for those who received PMRT $(n=130)$ and those who did not $(n=584)$. As expected, many factors showed an uneven distribution, including histologic type, RM, LVI, HG, and the number of positive LNs.

\section{Patterns of the first failure}

The patterns of the first failure are shown in Table 3. Most patients who experienced recurrence had distant metastasis $(82 \%, 54 / 66)$. Isolated local recurrence and regional recurrence were detected in three and nine patients, respectively.

\section{Prognostic factors for survival}

The median follow-up duration was 69 months (range, 1 to 114 months). The 5-year LRRFS, DFS, and OS rate were $97 \%, 94 \%$, and 98\%, respectively, in patients who received PMRT (PMRT [+]). The corresponding figures were 96\%, $90 \%$, and $96 \%$, respectively, in patients who did not receive PMRT (PMRT [-]). PMRT did not significantly reduce the 5-year LRR rate (3.1\% vs. $4.3 \%, \mathrm{p}=0.571)$. The $\mathrm{HG}$ and molecular subtype were statistically significant prognostic factors for LRRFS and DFS upon univariable analysis (Table 4). Upon multivariable analysis, only the HG remained statistically significant. For OS, the HG showed marginal significance in both univariable and multivariable analyses.

\section{Subgroup analysis of high HG disease}

Because HG was the most important prognostic factor for survival in this study population, we conducted a subgroup analysis of HG $3(n=305)$. Although the p-value was > 0.05,
PMRT (+) patients showed better survival by the KaplanMeier curve, especially for the DFS ( $\mathrm{p}=0.081$ ) (Fig. 1).

\section{Propensity score matching}

Propensity score matching was conducted to evaluate the impact of PMRT while minimizing the effects of other prognostic factors. After we performed the propensity score matching process, all factors were well balanced between treatment groups (S1 Table). There was no difference in the LRRFS, DFS, and OS between PMRT (-) and PMRT (+) patients (Fig. 2, S2 Table).

\section{Discussion}

The purpose of this study was to evaluate the effects of PMRT on the LRRFS, DFS, and OS in pN1 patients treated with modern systemic chemotherapy after MRM. Several randomized studies have shown the benefit of PMRT in pT12N1 patients who were treated with CMF. However, the role of PMRT in pT1-2N1 patients treated with modern taxanebased chemotherapy is still controversial.

The addition of taxane to AC resulted in significant improvement in the DFS in several studies. In the National Surgical Adjuvant Breast and Bowel Project (NSABP) B-28 trial [8], the addition of taxane to AC significantly reduced the hazard for a DFS event by $17 \%$, with a 5 -year DFS of $76 \%$ and $72 \%$ for AC-T and AC, respectively. Another study by the Cancer and Leukemia Group B (CALGB) 9334 [9] showed similar results. Despite delays in radiotherapy during addi- 


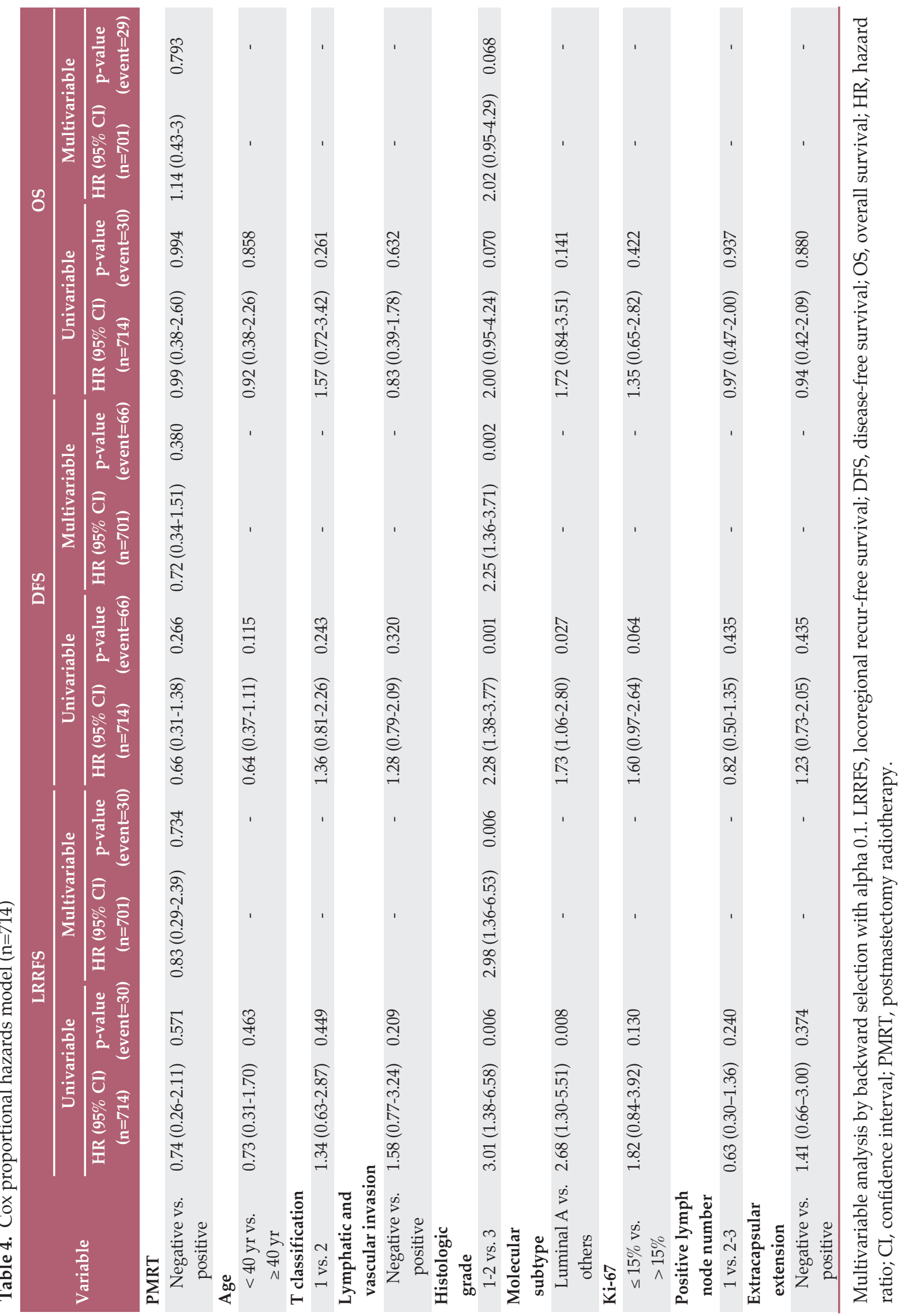



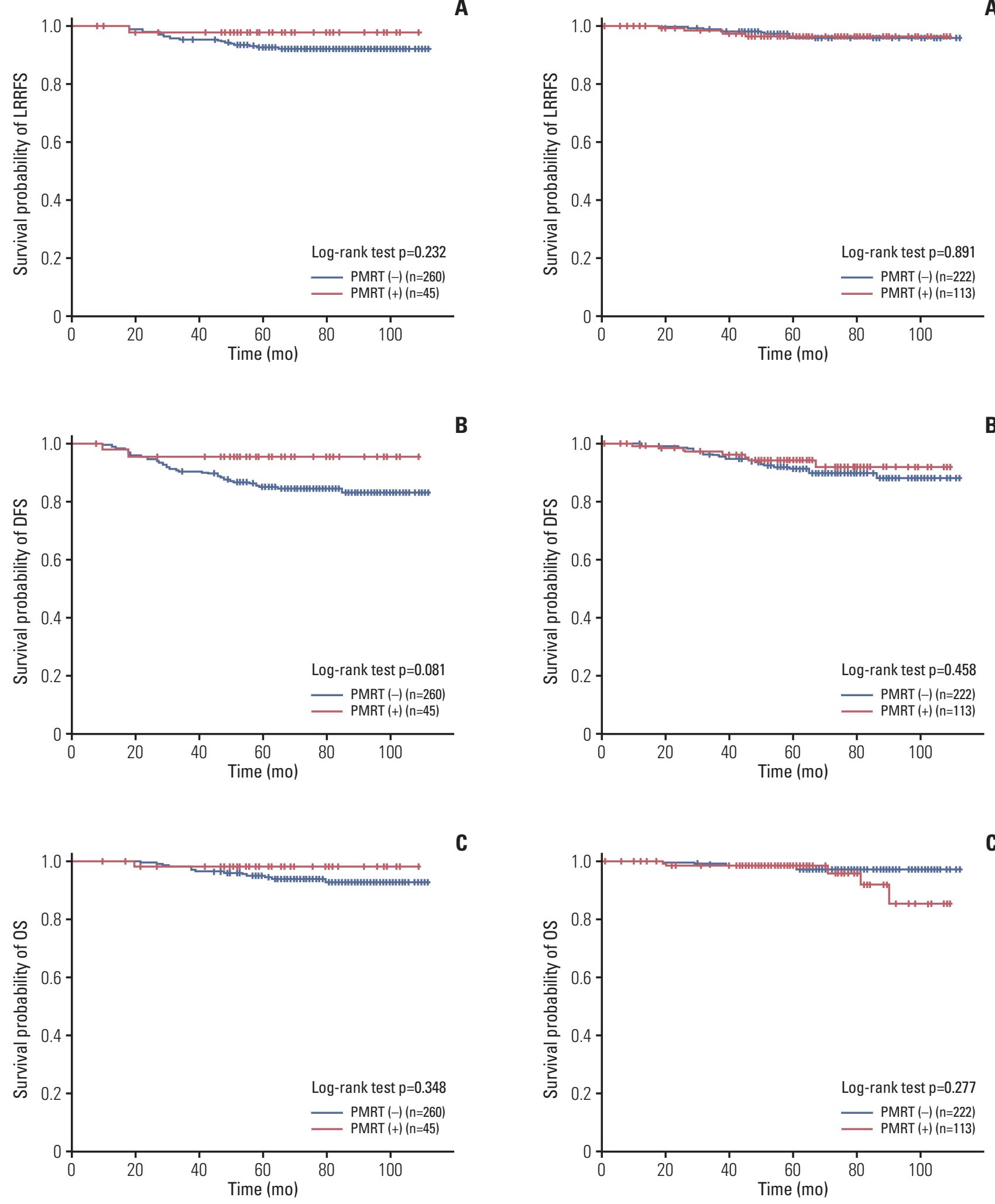

Fig. 1. Survival rates of histologic grade 3 patients $(n=305)$. Loco-regional recurrence-free survival (LRRFS) (A), disease-free survival (DFS) (B), and overall survival (OS) (C). PMRT, postmastectomy radiotherapy.

Fig. 2. Survival rates after propensity score matching $(\mathrm{n}=335)$. Loco-regional recurrence-free survival (LRRFS) (A), disease-free survival (DFS) (B), and overall survival (OS) (C). PMRT, postmastectomy radiotherapy. 
tional chemotherapy, adjuvant AC-T afforded better local control than $\mathrm{AC}$ alone in patients treated with breast-conserving therapy. The 5-year cumulative incidence of isolated LRR was $9.7 \%$ in the AC arm and $3.7 \%$ in the AC-T arm $(\mathrm{p}=0.04)$, while that of LRR as any component of failure was $12.9 \%$ versus $6.1 \%$, respectively ( $\mathrm{p}=0.04$ ). A meta-analysis [10] of long-term outcomes among 100,000 women in 123 randomized trials confirmed the benefit of taxane, with the addition of four separate cycles of taxane to a fixed anthracycline-based control regimen reducing breast cancer mortality.

A previous retrospective study [11] analyzed the LRR rates during an early era (1978-1997) and a later era (2000-2007). The later era was thought to represent periods of routine use of sentinel LN surgery, taxane-based chemotherapy, and aromatase inhibitors. PMRT did not appear to benefit patients treated in the later cohort (5-year LRR rates of 2.8\% without PMRT and $4.2 \%$ with PMRT, $\mathrm{p}=0.48$ ), but it did reduce the rate of LRR in the early era cohort (5-year rates of 9.5\% without PMRT and $3.4 \%$ with PMRT, $\mathrm{p}=0.028$ ). The most significant factor predictive of LRR for patients who did not receive PMRT was the era in which the patient was treated.

A recent study [12] revealed similar findings. Specifically, later-era (2004-2011) patients had a significantly lower 5-year LRR than early-era patients (1998-2011) (3.2\% vs. $10.3 \%$, $\mathrm{p}<0.001)$. In the later-era patients, PMRT did not significantly reduce the 5 -year LRR rate ( $1 \%$ vs. $3.8 \%, \mathrm{p}=0.206)$, while it did improve the 5 -year DFS rate $(96.1 \%$ vs. $87.5 \%$, $\mathrm{p}=0.015$ ). Although more patients in the late era received $\mathrm{AC} \pm \mathrm{T}$ chemotherapy than in the early era, $15 \%$ in the late era were still treated with CMF.

The strength of our study population is the homogeneity of the taxane-based chemotherapy regimen. In our study, PMRT did not significantly reduce the 5-year LRR rate. PMRT also had no significant impact on DFS, LRRFS, or OS. Even after propensity score matching, PMRT had no significant impact on survival. Risk factors for LRR in pT1-2N1 patients who have undergone MRM have been evaluated in many studies. However, the results have not been consistent. The main reason for this inconsistency is the different chemotherapy regimens. Age, tumor size, premenopausal status, the number of positive LNs, and the number of dissected LNs were significant predictors for LRR based on five NSABP randomized clinical trials [13]. In these five randomized trials, the chemotherapy was AC. In another study [14], LVI, ECE, and the number and level of involved axillary nodes were the prognostic factors associated with SCL LN recurrence. In that study, the chemotherapy regimen was CMF.

Because breast cancer is associated with highly heterogeneous tumors, several studies have analyzed the association between molecular subtypes of breast cancer and LRR. Wen et al. [15] showed that, when compared with the luminal A subtype, the HER2-enriched and basal-like subtypes were associated with significantly higher 5-year LRR rates, lower 5-year LRRFS rates, and poorer 5-year breast cancer-specific survival rates in pT1-2N1M0 breast cancer patients who did not undergo PMRT. That study population was treated between 1998 and 2010, and the chemotherapy regimens were not mentioned. In a different study [16], researchers evaluated the impact of molecular subtype on LRR in mastectomy patients with pT1-2N1. Only age $(\leq 50$ years, $p=0.003)$ and presence of LVI ( $p=0.0003$ ) were predictive of $\mathrm{LRR}$, while molecular subtype was not $(\mathrm{p}=0.38)$. Additionally, only $85 \%$ of the patients received systemic chemotherapy, while $67 \%$ received an anthracycline/taxane-based regimen.

A recent study [17] that included patients who received an anthracycline-based $(45 \%)$ or taxane-based $(3 \%)$ regimen, or both $(29 \%)$, revealed that young age ( $\leq 40$ years), a tumor larger than $3 \mathrm{~cm}$, and the presence of extensive intraductal components were significant risk factors for LRR. Another recent study [18] reported that LVI+ results, HG 3, and the nonluminal A subtype were poor prognostic factors for pT1$2 \mathrm{~N} 1$ breast cancer patients who underwent breast conserving surgery or MRM, received adjuvant taxane-based chemotherapy, and did not receive elective nodal irradiation.

In our study, only HG showed statistical significance for predicting LRRFS and DFS. To the best of our knowledge, this is the first study to report prognostic factors for survival in pT1-2N1 patients who have undergone MRM and received taxane-based chemotherapy. Upon subgroup analysis of HG 3 patients, PMRT showed a marginal benefit for DFS. Because of the small number of patients and events in this subgroup analysis, we could not sufficiently show the role of PMRT in HG 3 patients. Our findings regarding the prognostic value of $\mathrm{HG}$ are consistent with those of a previous study that investigated the impact of PMRT in modern systemic therapy [19]. Specifically, that study found that HG 3 and ECE were significant risk factors for LRR. In the present study, the status of ECE was unknown in 112 patients. Considering that ECE is an important prognostic factor for LRR, this could have affected the results of our study.

This study had the intrinsic limitations of a retrospective study. First, because there were no standard guidelines for adjuvant radiation in pT1-2N1 patients, and PMRT was performed according to each physician's preference and institutional policy, the patient characteristics between the PMRT $(-)$ and PMRT (+) groups were imbalanced. We conducted propensity score matching to overcome these uneven distributions. However, after propensity score matching, the number of patients and events became smaller, which reduced the power to show the impact of PMRT. Second, SCL and IMN radiotherapy was only conducted in $99(76.2 \%, 99 / 130)$ 
and $61(46.9 \%, 61 / 130)$ patients, respectively, in the PMRT $(+)$ group. Accordingly, it is possible that incomplete coverage of the regional LN area counteracted the benefits of PMRT.

PMRT did not significantly reduce the 5-year LRR rate, and it had no significant impact on the LRRFS, DFS, or OS. PMRT (+) showed a marginal benefit for DFS in HG 3 patients. Randomized studies are needed to confirm the benefit of PMRT in high risk patients, such as those with HG 3.

\section{Electronic Supplementary Material}

Supplementary materials are available at Cancer Research and Treatment website (http://www.e-crt.org).

\section{Conflicts of Interest}

Conflict of interest relevant to this article was not reported.

\section{Author Details}

${ }^{1}$ Research Institute and Hospital, National Cancer Center, Goyang, ${ }^{2}$ Samsung Medical Center, Sungkyunkwan University School of Medicine, Seoul, ${ }^{3}$ Biometric Research Branch, National Cancer Center, Goyang, ${ }^{4}$ Department of Radiation Oncology, Seoul National University College of Medicine, Seoul, ${ }^{5}$ Department of Radiation Oncology, Hallym University Dongtan Sacred Heart Hospital, Hwaseong, 'Department of Radiation Oncology, Ewha Womans University Mokdong Hospital, Ewha Womans University School of Medicine, Seoul, 'Department of Radiation Oncology, Yonsei Cancer Center, Yonsei University College of Medicine, Seoul, ${ }^{8}$ Department of Radiation Oncology, Chonnam National University Medical School, Gwangju, ${ }^{9}$ Department of Radiation Oncology, St. Vincent's Hospital, College of Medicine, The Catholic University of Korea, Suwon, ${ }^{10}$ Department of Radiation Oncology, Keimyung University Dongsan Medical Center, Keimyung University School of Medicine, Daegu, ${ }^{11}$ Department of Radiation Oncology, Ajou University School of Medicine, Suwon, ${ }^{12}$ Department of Radiation Oncology, Dong-A University Hospital, Dong-A University School of Medicine, Busan, ${ }^{13}$ Department of Radiation Oncology, Chonbuk National University Medical School, Jeonju, ${ }^{14}$ Department of Radiation Oncology, Wonju Severance Christian Hospital, Wonju, Korea

\section{References}

1. National Comprehensive Cancer Network. NCCN clinical practice guidelines in oncology: breast cancer, version 2. Fort Washington, PA: National Comprehensive Cancer Network; 2016.

2. Ragaz J, Olivotto IA, Spinelli JJ, Phillips N, Jackson SM, Wilson $\mathrm{KS}$, et al. Locoregional radiation therapy in patients with highrisk breast cancer receiving adjuvant chemotherapy: 20-year results of the British Columbia randomized trial. J Natl Cancer Inst. 2005;97:116-26.

3. Overgaard M, Nielsen HM, Overgaard J. Is the benefit of postmastectomy irradiation limited to patients with four or more positive nodes, as recommended in international consensus reports? A subgroup analysis of the DBCG 82 b\&c randomized trials. Radiother Oncol. 2007;82:247-53.

4. EBCTCG (Early Breast Cancer Trialists' Collaborative Group), McGale P, Taylor C, Correa C, Cutter D, Duane F, et al. Effect of radiotherapy after mastectomy and axillary surgery on 10-year recurrence and 20-year breast cancer mortality: metaanalysis of individual patient data for 8135 women in 22 randomised trials. Lancet. 2014;383:2127-35.

5. Early Breast Cancer Trialists' Collaborative Group (EBCTCG). Effects of chemotherapy and hormonal therapy for early breast cancer on recurrence and 15-year survival: an overview of the randomised trials. Lancet. 2005;365:1687-717.

6. De Laurentiis M, Cancello G, D'Agostino D, Giuliano M, Gior- dano A, Montagna E, et al. Taxane-based combinations as adjuvant chemotherapy of early breast cancer: a meta-analysis of randomized trials. J Clin Oncol. 2008;26:44-53.

7. Mackey JR, Martin M, Pienkowski T, Rolski J, Guastalla JP, Sami A, et al. Adjuvant docetaxel, doxorubicin, and cyclophosphamide in node-positive breast cancer: 10-year follow-up of the phase 3 randomised BCIRG 001 trial. Lancet Oncol. 2013;14:72-80.

8. Mamounas EP, Bryant J, Lembersky B, Fehrenbacher L, Sedlacek SM, Fisher B, et al. Paclitaxel after doxorubicin plus cyclophosphamide as adjuvant chemotherapy for node-positive breast cancer: results from NSABP B-28. J Clin Oncol. 2005;23:3686-96.

9. Sartor CI, Peterson BL, Woolf S, Fitzgerald TJ, Laurie F, Turrisi AJ, et al. Effect of addition of adjuvant paclitaxel on radiotherapy delivery and locoregional control of node-positive breast cancer: cancer and leukemia group B 9344. J Clin Oncol. 2005;23:30-40.

10. Early Breast Cancer Trialists' Collaborative Group (EBCTCG), Peto R, Davies C, Godwin J, Gray R, Pan HC, et al. Comparisons between different polychemotherapy regimens for early breast cancer: meta-analyses of long-term outcome among 100,000 women in 123 randomised trials. Lancet. 2012;379: 432-44.

11. McBride A, Allen P, Woodward W, Kim M, Kuerer HM, 
Drinka EK, et al. Locoregional recurrence risk for patients with T1,2 breast cancer with 1-3 positive lymph nodes treated with mastectomy and systemic treatment. Int J Radiat Oncol Biol Phys. 2014;89:392-8.

12. Chang JS, Lee J, Kim KH, Sohn JH, Kim SI, Park BW, et al. Do recent advances in diagnostic and therapeutic procedures negate the benefit of postmastectomy radiotherapy in $\mathrm{N} 1$ patients with a low risk of locoregional recurrence? Medicine (Baltimore). 2015;94:e1259.

13. Taghian A, Jeong JH, Mamounas E, Anderson S, Bryant J, Deutsch $\mathrm{M}$, et al. Patterns of locoregional failure in patients with operable breast cancer treated by mastectomy and adjuvant chemotherapy with or without tamoxifen and without radiotherapy: results from five National Surgical Adjuvant Breast and Bowel Project randomized clinical trials. J Clin Oncol. 2004;22:4247-54.

14. Yu JI, Park W, Huh SJ, Choi DH, Lim YH, Ahn JS, et al. Determining which patients require irradiation of the supraclavicular nodal area after surgery for N1 breast cancer. Int J Radiat Oncol Biol Phys. 2010;78:1135-41.

15. Wen G, Zhang JS, Zhang YJ, Zhu YJ, Huang XB, Guan XX. Pre- dictive value of molecular subtyping for locoregional recurrence in early-stage breast cancer with N1 without postmastectomy radiotherapy. J Breast Cancer. 2016;19:176-84.

16. Moo TA, McMillan R, Lee M, Stempel M, Ho A, Patil S, et al. Impact of molecular subtype on locoregional recurrence in mastectomy patients with T1-T2 breast cancer and 1-3 positive lymph nodes. Ann Surg Oncol. 2014;21:1569-74.

17. Lai SF, Chen YH, Kuo WH, Lien HC, Wang MY, Lu YS, et al. Locoregional recurrence risk for postmastectomy breast cancer patients with T1-2 and one to three positive lymph nodes receiving modern systemic treatment without radiotherapy. Ann Surg Oncol. 2016;23:3860-9.

18. Yu JI, Park W, Choi DH, Huh SJ, Nam SJ, Kim SW, et al. Prognostic modeling in pathologic N1 breast cancer without elective nodal irradiation after current standard systemic management. Clin Breast Cancer. 2015;15:e197-204.

19. Tendulkar RD, Rehman S, Shukla ME, Reddy CA, Moore H, Budd GT, et al. Impact of postmastectomy radiation on locoregional recurrence in breast cancer patients with 1-3 positive lymph nodes treated with modern systemic therapy. Int J Radiat Oncol Biol Phys. 2012;83:e577-81. 